\title{
Development of events detector for monitoring cryoseisms in upper soils
}

\author{
N. Afonin ${ }^{1}$ and E. Kozlovskaya ${ }^{1,2}$ \\ ${ }^{1}$ Oulu Mining School, POB-3000, FIN-90014, University of Oulu, nikita.afonin@oulu.fi \\ ${ }^{2}$ Geological Survey of Finland, P.O. Box 96, FI-02151, Espoo
}

\begin{abstract}
In this article, we describe the first results of the development of the seismic events detector with an artificial neural network (ANN) based identification. Such a detector is necessary for studying seismic events induced by soil freezing that can be hazardous for urban and mining infrastructures. We used the data of about 300 such seismic events recorded by seismic station OUL of Northern Finland Seismological Network for testing the detector and neural network learning. We processed about two months of continuous data and found out, that in some cases the number of detected and identified seismic events per day depends on air temperature variation.
\end{abstract}

\section{INTRODUCTION}

For the solution of some scientific and applied problems, analysis of huge amount of seismic data is necessary. One of such problems is investigation of an impact of extreme weather changes to water-saturated shallow layers of the subsurface. Weather extremes such as rapid temperature decrease can result in so-called "frost quakes" or cryoseisms resulting from cracking of soil and rock that occurs when water in rock has suddenly frozen and expanded. As cryoseisms can be hazardous for industrial and civil objects, their monitoring and analysis is necessary to increase the safety of these objects.

One of the important tasks in studying cryoseisms is development of efficient data processing routine capable to separate cryoseisms from other seismic events and noise. Nowadays artificial neural networks (ANNs) are used in earthquake seismology for development of procedures for classification of seismic events. Examples are classification of recorded events into regional, local, teleseismic earthquakes or industrial blasts (Scarpetta et al., 2005), detection of low-magnitude events (Kislov et al., 2012) etc. Nevertheless, there are several reasons why ANNs are still not widely used. They are: difficult and long learning process, the necessity of taking into account characteristics of each seismic station of the monitoring network, difficulties in evaluation of quality and reliability of probabilistic classification results (Kislov and Gravirov, 2017). Because cryoseisms are present in seismograms as strong impulses with high signal-to-noise ratio, we applied an "STA/LTA" algorithm for their detection and simple neural network for their classification using selected characteristics of the records (length, central frequencies, etc.).

\section{DESCRIPTION OF THE DATA ANALYSIS}

We used continuous data recorded by seismic station OUL since the end of November, 2015 to the end of February, 2016. This time interval was selected because of the strong 
cryoseismic event in Talvikangas district of Oulu on 06.06.2016 that caused damage to road surface and basements of buildings. During this day, many seismic events with similar waveforms were recorded by the OUL station. Assuming that these events were also caused by soil freezing, we used them as a learning sample for the neural network.

Analysis of these events has shown that most of them have many similarities in selected records characteristics (central frequencies, duration etc.) with the major event and with each other. We applied these identification criteria to events detected during other time intervals and compare the number of events per day with air temperature (Figure 1)

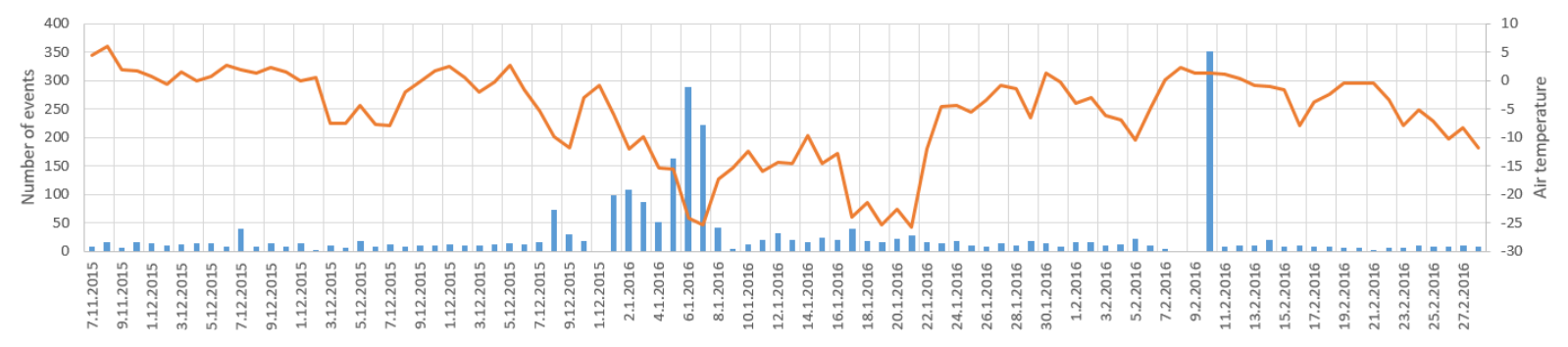

Figure 1: Dependence between air temperature and number of impulses per day.

As one can see in Figure 1, the number of events is highest since 1.01.2016 to 8.01.2016 and another peak in observed on 10.02.2016. In January, the increase in the number of events is most probably caused by the strong gradient of air temperature and hence fast soil freezing. Nevertheless, the huge number of impulses on 10.02.2016 is apparently not depended on the air temperature gradient. This may be caused by joint impact of atmosphere condition changes and hydrogeological processes in the subsurface, which requires further investigation.

\section{CONCLUSION}

We developed an efficient algorithm based on STA/LTA for detection and simple neural network for separation of cryoseisms from other seismic events. Comparison of results with air temperature variations partially confirms the detection results. Nevertheless, number of seismic events not always has correlation with air temperature, suggesting that cryoseisms can be caused by more complicated interaction between atmosphere and hydrological processes in the shallow subsurface.

\section{ACKNOWLEDGEMENTS}

This work is part of project "Impact of extreme weather events in the Arctic on technological systems, critical facilities and urban environment" funded by KVANTUM Institute of the University of Oulu.

\section{REFERENCES}

Scarpetta, S., F. Giudicepietro, E.C. Ezin, S. Petrosino, E. Del Pezzo, M. Martini and M. Marinaro, 2005. Automatic classification of seismic signals at Mt. Vesuvius Volcano, Italy, using neural networks. Bull. Seismol. Soc. Am., 95, 185-196. 
Kislov, K.V., F.E. Vinberg, A.F. Kushnir and V.V. Gravirov, 2012. Early warning system and man-made noise. Proceedings of the IV International Conference Problems of Cybernetics and Informatics, Baku, Azerbaijan, 52-55.

Kislov, K.V. and V.V. Gravirov, 2017. Using of artificial neural networks for classification of noisy seismic signals. Seismic instruments, 52, 46-64. 